ESAIM: PROCEEDINGS AND SURVEYS, September 2019, Vol. 66, p. 42-60

Philippe Helluy, Jean-Marc Hérard and Nicolas Seguin Editors

\title{
CLOSURE CONDITIONS FOR A ONE TEMPERATURE NON-EQUILIBRIUM MULTI-COMPONENT MODEL OF BAER-NUNZIATO TYPE
}

\author{
M. HANTKE ${ }^{1}$ AND S. MÜLLER ${ }^{2}$
}

\begin{abstract}
A class of non-equilibrium models for compressible multi-component fluids is investigated. These models are subject to the choice of interfacial pressures and interfacial velocity as well as relaxation terms for velocity, pressure and chemical potentials. Sufficient conditions are derived for these quantities that ensure meaningful physical properties such as a non-negative entropy production and thermodynamical stability as well as mathematical properties such as hyperbolicity. For the relaxation of chemical potentials a three-component model gas-water-vapor is considered.
\end{abstract}

\section{INTRODUCTION}

In the literature there are numerous two-phase mixture models available. A well-known example are BaerNunziato type models, cf. [3]. These can be derived by the ensemble averaging procedure of Drew [5]. A comprehensive introduction to these models can be found in the classical book of Drew and Passman [6]. Since each component of the mixture is governed by its own set of balance equations, these models are typically very expensive to solve numerically. While non-equilibrium models are required for plasma flows and geothermics, for many multiphase applications, see [16] for more details, reduced models are appropriate having diminished complexity and, thus, saving computational time.

Originally, Baer and Nunziato [3] proposed a two-phase model for detonation waves in granular explosives. This model is a full non-equilibrium model, i.e., each component has its own pressure, velocity and temperature and is governed by its own set of fluid equations. There are simplified models available in the literature that can be derived from the above general model by assuming zero relaxation times. In [17] a hierarchy of such models was derived by considering the asymptotic limit for velocity, pressure, temperature and Gibbs free energy. These models are classified by the number of equations in case of two phases in one space dimension. For instance, a six-equation model with a single velocity was originally derived by assuming a zero velocity relaxation time. It consists of one volume fraction equation, two mass balance equations, a mixture momentum equation and two energy equations. Assuming zero relaxation time for both the velocity and the pressure a five-equation model with mechanical equilibrium, i.e., single velocity and single pressure, is deduced in the asymptotic limit. This model is composed of two mass equations, a mixture momentum equation and a mixture energy equation. The four-equation model has a single velocity, single pressure and also single temperature coinciding with the single-fluid reactive Euler equations. While the three-equation model is the system of Euler equations. It has single velocity, pressure, temperature, and also single chemical potential, i.e., it is in full equilibrium.

All the aforementioned reduced Baer-Nunziato type models assume velocity equilibrium. Some of these reduced models suffer from short-comings. For instance, conservation of energy might be violated or the system

${ }^{1}$ Institut für Mathematik, Martin-Luther-Universität Halle - Wittenberg, Theodor-Lieser-Str. 5, D-06120 Halle, Germany

${ }^{2}$ Institut für Geometrie und Praktische Mathematik, RWTH Aachen University, Templergraben 55, D-52056 Aachen, Germany

(C) EDP Sciences, SMAI 2019

This is an Open Access article distributed under the terms of the Creative Commons Attribution License (http://creativecommons.org/licenses/by/4.0), which permits unrestricted use, distribution, and reproduction in any medium, provided the original work is properly cited. 
looses its hyperbolicity. A detailed discussion of these models is beyond the scope of this work. For this purpose the interested reader is referred to [26] and the references cited therein. A rather complete hierarchy of such models is investigated in [9] where, in particular, the subcharacteristic condition is verified.

A different hierarchy of models has been introduced in [16] where three classes are distinguished. The full non-equilibrium models are referred to as class-III models. Class-II models have a single temperature while class-I models have a single temperature as well as a single velocity.

Which of the models is applicable for a problem at hand strongly depends on the time scales over which a quantity relaxes to equilibrium. For instance, in [17] it is mentioned that neither thermal nor chemical equilibrium are justified for deflagration-to-detonation transition (DDT) problems. On the other hand, there is no argument against using class-II models for flows of homogeneous mixtures of gases or liquids.

To our knowledge there is no literature available considering class-I or class-II models in the context of BaerNunziato models. Therefore, in the present work we consider a reduced multi-component Baer-Nunziato type model that can be considered as a class-II model in the hierarchy of fluid mixture models introduced in [16]. Here mass and momentum balances for all components are formulated but only one energy balance for the mixture. A similar class-II model that is not of Baer-Nunziato type has been investigated in [4] and [13]. The basic variables in this class are the mass densities, the velocities and the mixture temperature. Phase transitions as well as the exchange of momentum and energy are modeled by relaxation terms.

The paper is organized as follows: In the first section we introduce the model. Thermodynamic quantities are related by an equation of state, given in Section 2. In the following Section 3 we verify hyperbolicity of the system. To close the system, the interfacial pressures, the interfacial velocity as well as the relaxation terms have to be modeled in a thermodynamic consistent manner. This will be discussed in Sections 4 and 5 . We summarize the results in a conclusion.

\section{Mathematical Model}

The multi-component flow is described by a non-equilibrium model where all components are present in each point of the space-time continuum. Each component $k=1, \ldots, K$ has density $\rho_{k}$, velocity $\boldsymbol{v}_{k}$ and pressure $p_{k}$, The amount of each component is determined by its volume fraction $\alpha_{k}$ subject to the saturation constraint

$$
\sum_{k=1}^{K} \alpha_{k}=1, \quad \alpha_{k} \in(0,1)
$$

i.e., the components are considered to be immiscible. For all components of the mixture mass and momentum balances are formulated but only one energy balance for the mixture:

$$
\begin{aligned}
& \partial_{t}\left(\alpha_{k} \rho_{k}\right)+\nabla \cdot\left(\alpha_{k} \rho_{k} \boldsymbol{v}_{k}\right)=S_{\alpha \rho, k} \\
& \partial_{t}\left(\alpha_{k} \rho_{k} \boldsymbol{v}_{k}\right)+\nabla \cdot\left(\alpha_{k} \rho_{k} \boldsymbol{v}_{k} \boldsymbol{v}_{k}^{T}+\alpha_{k} p_{k} \boldsymbol{I}\right)=-\sum_{l=1}^{K} P_{k, l} \nabla \alpha_{l}+\boldsymbol{S}_{\alpha \rho \boldsymbol{v}, k}, \\
& \left.\partial_{t}(\rho E)+\nabla \cdot\left(\sum_{k=1}^{K} \alpha_{k} \rho_{k}\left(E_{k}+p_{k} / \rho_{k}\right)\right) \boldsymbol{v}_{k}\right)=0 .
\end{aligned}
$$

We do not take into account viscosity and heat conduction and we neglect effects due to surface tension and gravity. In our notation $E_{k}=e_{k}+\boldsymbol{v}_{k}^{2} / 2$ is the total specific energy with $e_{k}$ the specific internal energy of component $k$. These are related to the total energy density $\rho E$ of the mixture

$$
\rho E=\sum_{k=1}^{K} \alpha_{k} \rho_{k}\left(e_{k}+\frac{1}{2} \boldsymbol{v}_{k}^{2}\right)=\rho e+\frac{1}{2} \sum_{k=1}^{K} \alpha_{k} \rho_{k} \boldsymbol{v}_{k}^{2},
$$


where $E$ and $e$ denote the specific total and the specific internal energy, respectively. In addition, we define the mixture quantities for pressure, density and velocity

$$
p:=\sum_{k=1}^{K} \alpha_{k} p_{k}, \rho:=\sum_{k=1}^{K} \alpha_{k} \rho_{k}, \boldsymbol{v}:=\frac{1}{\rho} \sum_{k=1}^{K} \alpha_{k} \rho_{k} \boldsymbol{v}_{k} .
$$

The interfacial pressures $P_{k, l}$ account for different pressures at the phase interface between components $k$ and $l$. These are subject to the following constraints

$$
P_{k, k}=0, \quad \forall k=1, \ldots, K \quad \text { and } \quad P_{l}:=\sum_{k=1}^{K} P_{k, l}=\text { const } \quad \forall l=1, \ldots, K
$$

Note that $(1.5)_{1}$ can always be ensured by the saturation condition (1.1) replacing $P_{k, l}$ by $P_{k, l}-P_{k, k}$ whereas $(1.5)_{2}$ ensures that the mixture model can be written in conservative form. Because of these interfacial quantities the momentum equations $(1.2 \mathrm{~b})$ cannot be written in conservative form.

The source terms $S_{\alpha, k}, S_{\alpha \rho, k}$ and $\boldsymbol{S}_{\alpha \rho \boldsymbol{v}, k}$ on the right-hand sides of (1.2a), (1.2b) and (1.8) describe the relaxation process due to mass, momentum and volume fraction between the different components corresponding to the relaxation of velocity, pressure and chemical potentials, $\xi \in\{v, p, \mu\}$, i.e.,

$$
S_{\alpha, k}:=\sum_{\xi} S_{\alpha, k}^{\xi}, S_{\alpha \rho, k}:=\sum_{\xi} S_{\alpha \rho, k}^{\xi}, S_{\alpha \rho \boldsymbol{v}, k}:=\sum_{\xi} \boldsymbol{S}_{\alpha \rho \boldsymbol{v}, k}^{\xi} .
$$

These depend on the specific components at hand as discussed in Section 5. In order to ensure conservation of mass, momentum and energy of the mixture, the relaxation terms (1.6) have to satisfy the conservation constraints

$$
\sum_{k=1}^{K} S_{\alpha, k}^{\xi}=0, \quad \sum_{k=1}^{K} S_{\alpha \rho, k}^{\xi}=0, \quad \sum_{k=1}^{K} \boldsymbol{S}_{\alpha \rho \boldsymbol{v}, k}^{\xi}=\mathbf{0} \quad \forall \xi \in\{v, p, \mu\} .
$$

The evolution of the volume fractions is characterized by the non-conservative equation

$$
\partial_{t} \alpha_{k}+\boldsymbol{V}_{I} \cdot \nabla \alpha_{k}=S_{\alpha, k}
$$

with $\boldsymbol{V}_{I}$ the interfacial velocity. Due to the saturation condition (1.1) we only need $K-1$ equations. Without loss of generality we express $\alpha_{K}$ by the other volume fractions, i.e.,

$$
\alpha_{K}=1-\sum_{k=1}^{K-1} \alpha_{k}, \nabla \alpha_{K}=-\sum_{k=1}^{K-1} \nabla \alpha_{k}, S_{\alpha, K}=-\sum_{k=1}^{K-1} S_{\alpha, k} .
$$

The fluid equations (1.2) are supplemented by an equation of state for the phasic pressures and internal energies. Throughout this work we confine ourselves to simple mixtures, i.e.,

$$
p_{k}=p_{k}\left(\rho_{k}, T\right) \quad \text { resp. } \quad e_{k}=e_{k}\left(\rho_{k}, T\right)
$$

where the mixture temperature $T$ is implicitly given by the internal energy of the mixture $\rho e=\sum_{k=1}^{K} \alpha_{k} \rho_{k} e_{k}\left(\rho_{k}, T\right)$ resulting in $T=\tilde{T}\left(\rho e, \rho_{1}, \ldots, \rho_{K}, \alpha_{1}, \ldots, \alpha_{K-1}\right)$ where we employ (1.9).

For the verification of some physical and mathematical properties it will be helpful to rewrite the system of equations for the non-equilibrium model in terms of primitive quantities $\alpha_{k}, \rho_{k}, \boldsymbol{v}_{k}, T$. From the evolution 
equations $(1.2),(1.8)$ and the relation $T=\tilde{T}\left(\rho e, \rho_{1}, \ldots, \rho_{K}, \alpha_{1}, \ldots, \alpha_{K-1}\right)$ we deduce

$$
\begin{aligned}
\partial_{t} \rho_{k} & +\frac{\rho_{k}}{\alpha_{k}}\left(\boldsymbol{v}_{k}-\boldsymbol{V}_{I}\right) \cdot \nabla \alpha_{k}+\boldsymbol{v}_{k} \cdot \nabla \rho_{k}+\rho_{k} \nabla \cdot \boldsymbol{v}_{k}=S_{\rho, k}, \\
\partial_{t} \boldsymbol{v}_{k} & +\left(\nabla \boldsymbol{v}_{k}\right) \boldsymbol{v}_{k}+\frac{1}{\rho_{k}} \nabla p_{k}+\frac{p_{k}}{\alpha_{k} \rho_{k}} \nabla \alpha_{k}=-\frac{1}{\alpha_{k} \rho_{k}} \sum_{l=1}^{K} P_{k, l} \nabla \alpha_{l}+S_{\boldsymbol{v}, k}, \\
\partial_{t} T & +\left(\rho c_{v}\right)^{-1} \sum_{k=1}^{K}\left(-\rho_{k}^{2} \partial_{\rho_{k}} e_{k}\left(\boldsymbol{v}_{k}-\boldsymbol{V}_{I}\right)-\sum_{l=1}^{K} P_{l, k}\left(\boldsymbol{v}_{l}-\boldsymbol{V}_{I}\right)\right) \cdot \nabla \alpha_{k} \\
& +\left(\rho c_{v}\right)^{-1} \sum_{k=1}^{K} \alpha_{k}\left(p_{k}-\rho_{k}^{2} \partial_{\rho_{k}} e_{k}\right) \nabla \cdot \boldsymbol{v}_{k}+\left(\rho c_{v}\right)^{-1} \sum_{k=1}^{K} \alpha_{k} \rho_{k} c_{v, k} \boldsymbol{v}_{k} \cdot \nabla T=S_{T} .
\end{aligned}
$$

Here the velocity gradient is defined as $\nabla \boldsymbol{v}_{k}=\left(\nabla v_{k, 1}, \ldots, \nabla v_{k, d}\right)^{T}$. The corresponding relaxation terms are

$$
\alpha_{k} S_{\rho, k}=S_{\alpha \rho, k}-\rho_{k} S_{\alpha, k}, \alpha_{k} \rho_{k} S_{\boldsymbol{v}, k}=\boldsymbol{S}_{\alpha \rho \boldsymbol{v}, k}-S_{\alpha \rho, k} \boldsymbol{v}_{k}, \rho c_{v} S_{T}=S_{\rho e}+\sum_{k=1}^{K}\left(\rho_{k}^{2} \partial_{\rho_{k}} e_{k} S_{\alpha, k}-\left(e_{k}+\rho_{k} \partial_{\rho_{k}} e_{k}\right) S_{\alpha \rho, k}\right)
$$

So far, the model is not yet closed. For this purpose, we have to find closing conditions for the phasic pressures $p_{k}$ and the phasic energies $e_{k}$, the interfacial pressures $P_{k, l}$, the interfacial velocity $\boldsymbol{V}_{I}$ and the relaxation terms $S_{\alpha, k}, S_{\alpha \rho, k}$ and $\boldsymbol{S}_{\alpha \rho \boldsymbol{v}, k}$. In the following sections we will derive appropriate constraints. However, these will not specify a unique model but some options are still remaining for the choice of the interfacial velocity and the relaxation terms.

\section{Equation of State for Simple Mixtures}

In order to close the system of balance equations we have to determine the partial energies and partial pressures. For a simple mixture the partial quantities of a component only depend on its partial density and the temperature of the mixture. Let the constant material parameters $c_{v, k}, \gamma_{k}, \pi_{k}$ and $q_{k}$ denoting the specific heat capacity at constant volume, the adiabatic exponent, the minimal pressure and the heat of formation of component $k$, respectively, chosen such that $c_{v, k}>0, \pi_{k} \geq 0, q_{k} \in \mathbb{R}$ and $\gamma_{k}>1$. Let further $\rho_{k 0}$ be some reference density at reference temperature $T_{0}$. Then the stiffened gas EoS for component $k$ is specified. It holds

$$
\begin{aligned}
e_{k} & =c_{v, k} T+\pi_{k} / \rho_{k}+q_{k} \\
\mu_{k} & =-c_{v, k} T \ln \frac{T}{T_{0}}+q_{k}\left(1-\frac{T}{T_{0}}\right)+\left(\gamma_{k}-1\right) c_{v, k} T \ln \frac{\rho_{k}}{\rho_{k 0}}+\left(\gamma_{k}-1\right) c_{v, k} T-\frac{\pi_{k}}{\rho_{k 0}} \frac{T}{T_{0}} \\
p_{k} & =\rho_{k}\left(\gamma_{k}-1\right) c_{v, k} T-\pi_{k} \\
s_{k} & =c_{v, k}+c_{v, k} \ln \frac{T}{T_{0}}+q_{k} \frac{1}{T_{k 0}}-\left(\gamma_{k}-1\right) c_{v, k} \ln \frac{\rho_{k}}{\rho_{k 0}}-\frac{\pi_{k}}{\rho_{k 0}} \frac{1}{T_{0}} \\
a_{k}^{2} & =c_{v, k} T\left(\gamma_{k}-1\right) \gamma_{k}=\gamma_{k} \bar{a}_{k}^{2},
\end{aligned}
$$

where $\bar{a}_{k}$ denotes the isothermal speed of sound of component $k$, that can be written as

$$
a_{k}^{2}=\bar{a}_{k}^{2}+\frac{T}{\rho_{k}^{2} c_{v, k}}\left(\frac{\partial p_{k}}{\partial T}\right)^{2}
$$


For the mixture temperature we then deduce

$$
T=\frac{\rho e-\pi-\rho q}{\rho c_{v}} \quad \text { with } \quad \rho q:=\sum_{k=1}^{K} \rho_{k} q_{k} \quad, \quad \pi:=\sum_{k=1}^{K} \pi_{k} \quad \text { and } \quad \rho c_{v}:=\sum_{k=1}^{K} \rho_{k} c_{v, k}
$$

To ensure finite speeds of propagation, the sound speed $a_{k}$ and $\bar{a}_{k}$ have to be real numbers. For this reason the hyperbolicity conditions $a_{k}^{2} \geq 0$ and $\bar{a}_{k}^{2} \geq 0$ have to hold. Note that $a_{k}^{2} \geq \bar{a}_{k}^{2}$ provided that $c_{v, k}>0, \gamma_{k}>1$ and $T>0$.

Remark 1. For $\pi_{k}=0$ and $q_{k}=0$ the stiffened gas equation of state reduces to the ideal gas equation.

\section{Hyperbolicity}

Neglecting relaxation processes in the fluid equations (1.2) the model reduces to a homogeneous first order system. In the following we verify that the homogeneous system is hyperbolic, i.e., all eigenvalues of the Jacobian of the inviscid flux in any direction are real and there exists a basis of right (left) eigenvectors that spans the state space. This is subject to the constraint of non-resonance. Then all wave speeds are finite and the system may be locally decoupled. From a mathematical point of view, this property is helpful in the construction of numerical fluxes, in particular, Riemann solvers, reconstruction polynomials and limiters applied to characteristic variables.

To investigate hyperbolicity it is most convenient to consider the evolution equations (1.8) and (1.11) for the volume fractions, the mass densities, the velocities and the temperature, respectively, neglecting relaxation terms. For a non-reacting simple mixture the resulting system forms a quasi-conservative system

$$
\partial_{t} \boldsymbol{w}+\sum_{i=1}^{d} \boldsymbol{B}_{i}(\boldsymbol{w}) \partial_{x_{i}} \boldsymbol{w}=\mathbf{0}
$$

for the primitive variables $\boldsymbol{w}=\left(\alpha_{1}, \ldots, \alpha_{K-1}, \boldsymbol{w}_{1}^{T}, \ldots, \boldsymbol{w}_{K}^{T}, T\right)^{T}, \boldsymbol{w}_{k}=\left(\rho_{k}, \boldsymbol{v}_{k}^{T}\right)^{T}$ with $\boldsymbol{B}_{i}$ being block matrices

$$
\boldsymbol{B}_{i}:=\left(\begin{array}{ccccc}
V_{I, i} \boldsymbol{I}_{K-1} & & & & \\
\boldsymbol{A}_{1, i} & \boldsymbol{B}_{1, i} & & & \boldsymbol{b}_{1, i} \\
\vdots & & \ddots & & \vdots \\
\boldsymbol{A}_{K, i} & & & \boldsymbol{B}_{K, i} & \boldsymbol{b}_{K, i} \\
\boldsymbol{a}_{i}^{T} & \boldsymbol{c}_{1, i}^{T} & \cdots & \boldsymbol{c}_{K, i}^{T} & c_{i}
\end{array}\right)
$$

where the blocks are defined as

$$
\boldsymbol{A}_{k, i}:=\left(\begin{array}{c}
\boldsymbol{\alpha}_{k, i}^{T} \\
\boldsymbol{e}_{i} \boldsymbol{\beta}_{k}^{T}
\end{array}\right), \boldsymbol{B}_{k, i}:=\left(\begin{array}{cc}
v_{k, i} & \rho_{k} \boldsymbol{e}_{i}^{T} \\
\frac{\partial_{\rho_{k}} p_{k}}{\rho_{k}} \boldsymbol{e}_{i} & v_{k, i} \boldsymbol{I}_{d}
\end{array}\right), \boldsymbol{b}_{k, i}:=\left(\begin{array}{c}
0 \\
\frac{\partial_{T} p_{k}}{\rho_{k}} \boldsymbol{e}_{i}
\end{array}\right)=\frac{\rho c_{v}}{\alpha_{k} \rho_{k} T} \boldsymbol{c}_{k, i}, c_{i}:=\sum_{k=1}^{K} \frac{\alpha_{k} \rho_{k} c_{v, k}}{\rho c_{v}} v_{k i}
$$


Here $\boldsymbol{I}_{d}$ and $\boldsymbol{I}_{K-1}$ are the unit matrices in $\mathbb{R}^{d \times d}$ and $\mathbb{R}^{(K-1) \times(K-1)}$, respectively. The vectors $\boldsymbol{\alpha}_{k, i}, \boldsymbol{\beta}_{k}$ and $\boldsymbol{a}$ are defined by their components $l=1, \ldots, K-1$ as

$$
\begin{aligned}
\alpha_{k, i, l} & :=\frac{\rho_{k}}{\alpha_{k}}\left(v_{k, i}-V_{I, i}\right)\left(\delta_{i, l}^{T}\left(1-\delta_{k, K}\right)-\delta_{k, K}\right), \\
\beta_{k, l} & :=\frac{1}{\alpha_{k} \rho_{k}}\left(\left(P_{k, l}-p_{k}\right)\left(1-\delta_{k, l}\right)-\left(P_{k, K}-p_{k}\right)\left(1-\delta_{k, K}\right)\right), \\
a_{i, l} & :=\left(\rho c_{v}\right)^{-1}\left(\rho_{K}^{2} \partial_{\rho_{K}} e_{K}\left(v_{K, i}-V_{I, i}\right)-\rho_{l}^{2} \partial_{\rho_{l}} e_{l}\left(v_{l, i}-V_{I, i}\right)+\sum_{k=1}^{K}\left(P_{k, K}-P_{k, l}\right)\left(v_{k, i}-V_{I, i}\right)\right)
\end{aligned}
$$

with $\delta_{k, l}$ the Kronecker symbol. To verify hyperbolicity we have to check that for any direction $\boldsymbol{n} \in \mathbb{R}^{d},|\boldsymbol{n}|=1$, the projected matrix

$$
\boldsymbol{B}_{n}=\sum_{i=1}^{d} \boldsymbol{B}_{i} n_{i}
$$

has real eigenvalues and the corresponding eigenvectors form a basis for $\mathbb{R}^{K(d+2)}$. Introducing the normal velocities $v_{k, n}:=\boldsymbol{v}_{k} \cdot \boldsymbol{n}, V_{I, n}:=\boldsymbol{V}_{I} \cdot \boldsymbol{n}$ and the orthogonal block diagonal matrix $\boldsymbol{R}_{n}$ and the orthogonal matrix $\boldsymbol{O}_{n}$

$$
\boldsymbol{R}_{n}=\operatorname{diag}\left(\boldsymbol{I}_{K-1}, \boldsymbol{Q}_{n}, \ldots, \boldsymbol{Q}_{n}, 1\right), \boldsymbol{Q}_{n}=\operatorname{diag}\left(1, \boldsymbol{O}_{n}\right), \boldsymbol{O}_{n}=\left(\boldsymbol{n}, \boldsymbol{t}_{1}, \ldots, \boldsymbol{t}_{d-1}\right) \in \mathbb{R}^{d \times d}
$$

we can rewrite $\boldsymbol{B}_{n}$ as

$$
\tilde{\boldsymbol{B}}_{n}:=\boldsymbol{R}_{n}^{T} \boldsymbol{B}_{n} \boldsymbol{R}_{n}=\left(\begin{array}{cccccc}
V_{I, n} \boldsymbol{I}_{K-1} & & & & \\
\tilde{\boldsymbol{A}}_{1, n} & \tilde{\boldsymbol{B}}_{1, n} & & & \tilde{\boldsymbol{b}}_{1, n} \\
\vdots & & \ddots & & \vdots \\
\tilde{\boldsymbol{A}}_{K, n} & & & \tilde{\boldsymbol{B}}_{K, n} & \tilde{\boldsymbol{b}}_{K, n} \\
\boldsymbol{a}_{n}^{T} & \tilde{\boldsymbol{c}}_{1, n}^{T} & \cdots & \tilde{\boldsymbol{c}}_{K, n}^{T} & c_{n}
\end{array}\right)
$$

with entries

$$
\tilde{\boldsymbol{A}}_{k, n}:=\left(\begin{array}{c}
\boldsymbol{\alpha}_{k, n}^{T} \\
\boldsymbol{e}_{1} \boldsymbol{\beta}_{k}^{T}
\end{array}\right), \tilde{\boldsymbol{B}}_{k, n}:=\left(\begin{array}{cc}
v_{k, n} & \rho_{k} \boldsymbol{e}_{1}^{T} \\
\frac{\partial_{\rho_{k}} p_{k}}{\rho_{k}} \boldsymbol{e}_{1} & v_{k, n} \boldsymbol{I}_{d}
\end{array}\right), \tilde{\boldsymbol{b}}_{k, n}:=\left(\begin{array}{c}
0 \\
\frac{\partial_{T} p_{k}}{\rho_{k}} \boldsymbol{e}_{1}
\end{array}\right)=\frac{\rho c_{v}}{\alpha_{k} \rho_{k} T} \tilde{\boldsymbol{c}}_{k, n}, c_{n}:=\sum_{k=1}^{K} \frac{\alpha_{k} \rho_{k} c_{v, k}}{\rho c_{v}} v_{k, n}
$$

The vectors $\boldsymbol{\alpha}_{k, n}$ and $\boldsymbol{a}_{n}$ are defined by their components $l=1, \ldots, K-1$ as

$$
\begin{aligned}
\alpha_{k, n, l} & :=\frac{\rho_{k}}{\alpha_{k}}\left(v_{k, n}-V_{I, n}\right)\left(\delta_{k, l}\left(1-\delta_{k, K}\right)-\delta_{k, K}\right) \\
a_{k, n} & :=\left(\rho c_{v}\right)^{-1}\left(\rho_{K}^{2} \partial_{\rho_{K}} e_{K}\left(v_{K, n}-V_{I, n}\right)-\rho_{k}^{2} \partial_{\rho_{k}} e_{k}\left(v_{k, n}-V_{I, n}\right)+\sum_{i=1}^{K}\left(P_{i, K}-P_{i, k}\right)\left(v_{i, n}-V_{I, n}\right)\right) .
\end{aligned}
$$

Since $\boldsymbol{R}_{n}$ is an orthogonal matrix, the matrix $\tilde{\boldsymbol{B}}_{n}$ has the same eigenvalues as the matrix $\boldsymbol{B}_{n}$. A straightforward calculation gives

$$
\operatorname{det}\left(\tilde{\boldsymbol{B}}_{n}-\lambda \boldsymbol{I}\right)=\left(V_{I, n}-\lambda\right)^{K-1} \operatorname{det}\left(\overline{\boldsymbol{B}}_{n}-\lambda \boldsymbol{I}\right)=\left(V_{I, n}-\lambda\right)^{K-1}\left(\rho c_{v}\right)^{-1} \prod_{k=1}^{K}\left(v_{k, n}-\lambda\right)^{d-1} p_{K}(\lambda)
$$


with $\overline{\boldsymbol{B}}_{n}$ the reduced matrix determined from $\tilde{\boldsymbol{B}}_{n}$ by eliminating the first column and row, i.e.,

$$
\overline{\boldsymbol{B}}_{n}:=\left(\begin{array}{cccc}
\tilde{\boldsymbol{B}}_{1, n} & & & \tilde{\boldsymbol{b}}_{1, n} \\
& \ddots & & \vdots \\
& & \tilde{\boldsymbol{B}}_{K, n} & \tilde{\boldsymbol{b}}_{K, n} \\
\tilde{\boldsymbol{c}}_{1, n}^{T} & \cdots & \tilde{\boldsymbol{c}}_{K, n}^{T} & c_{n}
\end{array}\right)
$$

and the polynomial $p_{K}$ defined as

$$
p_{K}(\lambda):=\sum_{k=1}^{K} \alpha_{k} \rho_{k} c_{v, k}\left(v_{k, n}-\lambda\right)\left(\left(v_{k, n}-\lambda\right)^{2}-a_{k}^{2}\right) \prod_{j=1, j \neq k}^{K}\left(\left(v_{j, n}-\lambda\right)^{2}-\bar{a}_{j}^{2}\right) .
$$

Remark 2. In comparison to the Dreyer/Bothe model [4] investigated in [13] here the volume fraction enters in the polynomial (3.10) and $c_{v}$.

Remark 3. In case of a mixture of stiffened gases the sound speed $a_{k}$ tends to $\bar{a}_{k}$ for $T \rightarrow 0$ and $\gamma_{k} \rightarrow 1$. In the limit the roots of $p_{K}$ are given by

$$
\lambda_{k}=\sum_{k=1}^{K} \frac{\alpha_{k} \rho_{k} c_{v, k}}{\rho c_{v}} v_{k, n}, \quad \lambda_{k, \pm}=v_{k, n} \pm a_{k} .
$$

In general, it is hard, if not impossible, to determine explicitly all roots of the characteristic polynomial (3.10), and thus, it is not obvious that all eigenvalues are real. However, this follows directly if the matrix $\overline{\boldsymbol{B}}_{n}$ can be symmetrized using similarity transformations.

Theorem 1 (Existence of real eigenvalues). Let $\rho_{k}, \rho c_{v}, \partial_{T} p_{k} \neq 0$ (non-isothermal). If the state satisfies the condition

$$
\alpha_{k} \rho_{k} \frac{T}{\rho c_{v}}>0, \quad \frac{\partial_{\rho_{k}} p_{k}}{\rho_{k}^{2}}>0, \quad k=1, \ldots, K
$$

then all eigenvalues of $\overline{\boldsymbol{B}}_{n}$ are real.

Proof: To symmetrize $\overline{\boldsymbol{B}}_{n}$ using a similarity transformation, we multiply $\overline{\boldsymbol{B}}_{n}$ by the block diagonal matrix $\boldsymbol{D}:=\operatorname{diag}\left(\boldsymbol{D}_{1}, \ldots, \boldsymbol{D}_{K}, 1\right)$ with $\boldsymbol{D}_{k}:=\operatorname{diag}\left(\bar{\alpha}_{k}, \mathbf{1}+\left(\bar{\beta}_{k}-1\right) \boldsymbol{e}_{1}\right)$ and its inverse $\boldsymbol{D}^{-1}$ from the left and the right, respectively. Here we assume that the parameters $\bar{\alpha}_{k}$ and $\bar{\beta}_{k}$ are non-zero. Then the matrix $\check{\boldsymbol{B}}_{n}:=\boldsymbol{D} \overline{\boldsymbol{B}}_{n} \boldsymbol{D}^{-1}$ is symmetric if and only if

$$
\boldsymbol{D}_{k} \tilde{\boldsymbol{B}}_{k, n} \boldsymbol{D}_{k}^{-1}=\left(\boldsymbol{D}_{k} \tilde{\boldsymbol{B}}_{k, n} \boldsymbol{D}_{k}^{-1}\right)^{T}, \quad \tilde{\boldsymbol{c}}_{k, n} \boldsymbol{D}_{k}^{-1}=\boldsymbol{D}_{k} \tilde{\boldsymbol{b}}_{k, n}, \quad k=1, \ldots, K,
$$

or, equivalently,

$$
\bar{\alpha}_{k}^{-1} \bar{\beta}_{k} \frac{\partial_{\rho_{k}} p_{k}}{\rho_{k}}=\bar{\alpha}_{k} \bar{\beta}_{k}^{-1} \rho_{k}, \quad \bar{\beta}_{k}^{-1} \frac{\alpha_{k} T \partial_{T} p_{k}}{\rho c_{v}}=\bar{\beta}_{k} \frac{\partial_{T} p_{k}}{\rho_{k}}, \quad k=1, \ldots, K
$$

According to the assumptions (3.12) there exist non-vanishing real parameters $\bar{\alpha}_{k}$ and $\bar{\beta}_{k}$. Since a symmetric matrix has only real eigenvalues and these are invariant under the similarity transformation the assertion follows.

Remark 4. In comparison to the Dreyer/Bothe model [4] investigated in [13] here the volume fraction enters in the definition of $\bar{\beta}_{k}^{2}$.

Remark 5. For reasons of efficiency and robustness it is recommended to compute the eigenvalues of the matrix $\overline{\boldsymbol{B}}_{n}$ by applying a numerical eigenvalue solver to the symmetric matrix $\check{\boldsymbol{B}}_{n}=\boldsymbol{D} \overline{\boldsymbol{B}}_{n} \boldsymbol{D}^{-1}$ rather than by determining the roots of the characteristic polynomial $p_{K}$ defined by (3.10). 
Since now we know that all roots of the characteristic polynomial (3.8) are real, we determine the corresponding left and right eigenvectors. The following result is obvious and is given only for sake of completeness.

Proposition 1. There exist $(d-1)$-multiple eigenvalues $\lambda_{k, 0}=v_{k, n}, k=1, \ldots, K$ with corresponding linearly independent left and right eigenvectors $\boldsymbol{l}_{k, 0}^{i}$ and $\boldsymbol{r}_{k, 0}^{i}, i=1, \ldots, d-1$, determined by

$$
\boldsymbol{l}_{k, 0}^{i}=\boldsymbol{r}_{k, 0}^{i}=(\mathbf{0}_{K-1}^{T}, \underbrace{\mathbf{0}_{d+1}^{T}, \ldots, \mathbf{0}_{d+1}^{T}}_{k-1},\left(0, \boldsymbol{t}_{i}^{T}\right), \underbrace{\mathbf{0}_{d+1}^{T}, \ldots, \mathbf{0}_{d+1}^{T}}_{K-k}, 0)^{T}
$$

In particular, it holds $\nabla \boldsymbol{w} \lambda_{k, 0} \cdot \boldsymbol{r}_{k, 0}^{i}=0$, i.e., the corresponding characteristic fields are linearly degenerated, and, hence, correspond to contact discontinuities.

The eigenvectors determined in Proposition 1 correspond to the multi-dimensional case. The remaining eigenvectors can be determined basically from the one-dimensional case. For this reason we introduce the matrices and vectors $\tilde{\boldsymbol{A}}_{k, n}^{(1)}, \tilde{\boldsymbol{B}}_{k, n}^{(1)}, \tilde{\boldsymbol{b}}_{k, n}^{(1)}$ and $\tilde{\boldsymbol{c}}_{k, n}^{(1)}$ which are the one-dimensional counterparts to $\tilde{\boldsymbol{A}}_{k, n}, \tilde{\boldsymbol{B}}_{k, n}, \tilde{\boldsymbol{b}}_{k, n}$ and $\tilde{\boldsymbol{c}}_{k, n}$ defined in (3.6). In particular, it holds

$$
\tilde{\boldsymbol{A}}_{k, n}=\left(\begin{array}{c}
\tilde{\boldsymbol{A}}_{k, n}^{(1)} \\
\mathbf{0}_{(d-1) \times(K-1)}
\end{array}\right), \tilde{\boldsymbol{B}}_{k, n}=\left(\begin{array}{cc}
\tilde{\boldsymbol{B}}_{k, n}^{(1)} & \mathbf{0}_{d-1}^{T} \\
\mathbf{0}_{d-1} & v_{k, n} \boldsymbol{I}_{d-1}
\end{array}\right), \tilde{\boldsymbol{b}}_{k, n}=\left(\begin{array}{c}
\tilde{\boldsymbol{b}}_{k, n}^{(1)} \\
\mathbf{0}_{d-1}
\end{array}\right), \tilde{\boldsymbol{c}}_{k, n}=\left(\begin{array}{c}
\tilde{\boldsymbol{c}}_{k, n}^{(1)} \\
\mathbf{0}_{d-1}
\end{array}\right)
$$

To determine the remaining eigenvectors we proceed similar as in [13]. Since here we have to account in addition for the volume fractions, the proofs are not identical but the procedure is similar. Therefore, we omit the details of the derivation of the eigenvectors and only present the main results. First of all, we give the eigenvalues and eigenvectors of the matrix $\tilde{\boldsymbol{B}}_{k, n}$.

Lemma 1. The eigenvalues and corresponding eigenvectors of the matrices $\tilde{\boldsymbol{B}}_{k, n}, k=1, \ldots, K$, are determined by

$$
\begin{gathered}
\lambda_{k}=v_{k, n}(d-1) \text {-multiple }, \quad \lambda_{k, \pm}=v_{k, n} \pm \bar{a}_{k} \text { 1-multiple } \\
\boldsymbol{r}_{k, \pm}=\left( \pm \rho_{k} / \bar{a}_{k}, 1, \mathbf{0}_{d-1}\right)^{T}, \quad \boldsymbol{r}_{k, i}=\boldsymbol{e}_{i+2} \in \mathbb{R}^{d+1}, i=1, \ldots, d-1 .
\end{gathered}
$$

If the non-resonance conditions

$$
\begin{gathered}
\delta_{k}^{n}(\lambda):=v_{k, n}-\lambda \neq 0 \quad \text { and } \\
\sigma_{k}^{n}(\lambda):=\left(\delta_{k}^{n}(\lambda)\right)^{2}-\bar{a}_{k}^{2} \neq 0 \quad \forall k=1, \ldots, K
\end{gathered}
$$

hold, then the inverse $\tilde{\boldsymbol{B}}_{k, n}-\lambda \boldsymbol{I}$ exists and is given by

$$
\left(\tilde{\boldsymbol{B}}_{k, n}-\lambda \boldsymbol{I}\right)^{-1}=\left(\begin{array}{cc}
\left(\tilde{\boldsymbol{B}}_{k, n}^{(1)}-\lambda \boldsymbol{I}_{2}\right)^{-1} & \mathbf{0} \\
\mathbf{0} & \left(\delta_{k}^{n}(\lambda)\right)^{-1} \boldsymbol{I}_{d-1}
\end{array}\right)
$$

with

$$
\left(\tilde{\boldsymbol{B}}_{k, n}^{(1)}-\lambda \boldsymbol{I}_{2}\right)^{-1}=\frac{1}{\sigma_{k}^{n}(\lambda)}\left(\begin{array}{cc}
\delta_{k}^{n}(\lambda) & -\rho_{k} \\
-\bar{a}_{k}^{2} / \rho_{k} & \delta_{k}^{n}(\lambda)
\end{array}\right)
$$

The non-resonance conditions (3.17) and (3.18) essentially mean that $\lambda$ is not an eigenvalue of $\tilde{\boldsymbol{B}}_{k, n}$ and the eigenvalues of the matrices $\tilde{\boldsymbol{B}}_{k, n}^{(1)}, k=1, \ldots, K$ are no roots of the characteristic polynomial $p_{K}$ defined by (3.10). The latter does not hold true if $a_{k}=\bar{a}_{k}$. According to (2.2) this is the case for isothermal fluids, i.e., $p_{k}=p_{k}\left(\rho_{k}\right)$, that are of no interest here, cf. equation (1.10).

By means of the non-resonance condition (3.18) we can verify the following two lemmata. 
Lemma 2. Let be $\lambda \in \mathbb{R}$ such that the non-resonance condition (3.18) holds. Then the following relation is true:

$$
c_{n}-\lambda-\sum_{k=1}^{K}\left(\tilde{\boldsymbol{c}}_{k, n}^{(1)}\right)^{T}\left(\tilde{\boldsymbol{B}}_{k, n}^{(1)}-\lambda \boldsymbol{I}_{2}\right)^{-1} \tilde{\boldsymbol{b}}_{k, n}^{(1)}=\frac{p_{K}(\lambda)}{\kappa_{0}(\lambda) \rho c_{v}}
$$

with

$$
\kappa_{0}(\lambda):=\prod_{k=1}^{K} \sigma_{k}^{n}(\lambda) \neq 0
$$

Proof: First of all, the non-resonance condition (3.18) implies $\sigma_{k}^{n}(\lambda) \neq 0$ and, thus, $\kappa_{0} \neq 0$. Then by definitions of $\tilde{\boldsymbol{b}}_{k, n}^{(1)}$ and $\tilde{\boldsymbol{c}}_{k, n}^{(1)}$, see Eqns. (3.6) and (3.14), as well as Lemma 1 and Eqn. (3.20) it follows

$$
\tilde{\boldsymbol{c}}_{k, n}^{T}\left(\tilde{\boldsymbol{B}}_{k, n}-\lambda \boldsymbol{I}_{d+1}\right)^{-1} \tilde{\boldsymbol{b}}_{k, n}=\left(\tilde{\boldsymbol{c}}_{k, n}^{(1)}\right)^{T}\left(\tilde{\boldsymbol{B}}_{k, n}^{(1)}-\lambda \boldsymbol{I}_{2}\right)^{-1} \tilde{\boldsymbol{b}}_{k, n}^{(1)}=\left(\rho c_{v}\right)^{-1} \alpha_{k} \rho_{k} c_{v, k}\left(a_{k}^{2}-\bar{a}_{k}^{2}\right) \frac{\delta_{k}^{n}(\lambda)}{\sigma_{k}^{n}(\lambda)} .
$$

Finally, the assertion follows by definitions of $c_{n}$ and the polynomial $p_{K}$, see Eqns. (3.6) and (3.10), respectively.

Lemma 3. Let be $\lambda \in \mathbb{R}$ such that the non-resonance condition (3.18) holds. Then the following relation is true:

and, thus,

$$
\left(\tilde{\boldsymbol{A}}_{k, n}^{(1)}\right)^{T}\left(\tilde{\boldsymbol{B}}_{k, n}^{(1)}-\lambda \boldsymbol{I}_{2}\right)^{-T} \tilde{\boldsymbol{c}}_{k, n}^{(1)}=\frac{T}{\rho c_{v}} \frac{\alpha_{k} \partial_{T} p_{k}}{\rho_{k} \sigma_{k}^{n}(\lambda)}\left(\rho_{k} \delta_{k}^{n}(\lambda) \boldsymbol{\beta}_{k}-\bar{a}_{k}^{2} \boldsymbol{\alpha}_{k, n}\right)
$$

$$
\boldsymbol{z}(\lambda):=\sum_{k=1}^{K}\left(\tilde{\boldsymbol{A}}_{k, n}^{(1)}\right)^{T}\left(\tilde{\boldsymbol{B}}_{k, n}^{(1)}-\lambda \boldsymbol{I}_{2}\right)^{-T} \tilde{\boldsymbol{c}}_{k, n}^{(1)}=\frac{T}{\rho c_{v}} \sum_{k=1}^{K} \frac{\alpha_{k} \partial_{T} p_{k}}{\rho_{k} \sigma_{k}^{n}(\lambda)}\left(\rho_{k} \delta_{k}^{n}(\lambda) \boldsymbol{\beta}_{k}-\bar{a}_{k}^{2} \boldsymbol{\alpha}_{k, n}\right)
$$

Proof: By definition of $\tilde{\boldsymbol{A}}_{k, n}^{(1)}$ and $\tilde{\boldsymbol{c}}_{k, n}^{(1)}$ as the one-dimensional counterparts to $\tilde{\boldsymbol{A}}_{k, n}$ and $\tilde{\boldsymbol{c}}_{k, n}$ defined by (3.14) and (3.6), respectively, as well as Eqn. (3.20) it follows

$\tilde{\boldsymbol{A}}_{k, n}^{T}\left(\tilde{\boldsymbol{B}}_{k, n}-\lambda \boldsymbol{I}_{d+1}\right)^{-T} \tilde{\boldsymbol{c}}_{k, n}=\left(\tilde{\boldsymbol{A}}_{k, n}^{(1)}\right)^{T}\left(\tilde{\boldsymbol{B}}_{k, n}^{(1)}-\lambda \boldsymbol{I}_{2}\right)^{-T} \tilde{\boldsymbol{c}}_{k, n}^{(1)}=\frac{\alpha_{k}}{\rho c_{v} \sigma_{k}^{n}(\lambda) \partial_{T} p_{k}} \rho_{k} c_{v, k}\left(a_{k}^{2}-\bar{a}_{k}^{2}\right)\left(\rho_{k} \delta_{k}^{n} \boldsymbol{\beta}_{k}-\bar{a}_{k}^{2} \boldsymbol{\alpha}_{k, n}\right)$.

Applying (2.2) to the right-hand side we finally obtain the assertion.

By means of the above lemmata we can now determine the eigenvectors to the $(K-1)$-multiple eigenvalue $\lambda=V_{I, n}$.

Proposition 2. There exist $(K-1)$-multiple eigenvalues $\lambda_{i, 0}=V_{I, n}, i=1, \ldots, K-1$. If the non-resonance condition (3.18) holds for $\lambda_{i, 0}$ and $\lambda_{i, 0}$ is no root of the characteristic polynomial (3.10), then the corresponding right eigenvectors are determined by $\boldsymbol{r}_{I, i}^{T}=\left(\boldsymbol{r}_{I, i, 0}^{T}, \boldsymbol{r}_{I, i, 1}^{T}, \ldots, \boldsymbol{r}_{I, i, K}^{T}, r_{I, i}\right)$ with

$$
\begin{aligned}
\boldsymbol{r}_{I, i, 0} & =\boldsymbol{e}_{K-1, i}, \\
\boldsymbol{r}_{I, i, k} & =\frac{1}{\sigma_{k}^{n}}\left(\begin{array}{c}
-\delta_{k}^{n} \alpha_{k, n, i}+\rho_{k} \beta_{k, i} \\
\left(\bar{a}_{k}^{2} / \rho_{k} \alpha_{k, n, i}-\delta_{k}^{n} \beta_{k, i}\right) \boldsymbol{n}
\end{array}\right)+\frac{\partial_{T} p_{k}}{\rho_{k} \sigma_{k}^{n}}\left(\begin{array}{c}
\rho_{k} r_{I, i} \\
-\delta_{k}^{n} r_{I, i} \boldsymbol{n}
\end{array}\right), \\
r_{I, i} & =\frac{\rho c_{v} \kappa_{0}\left(V_{I, n}\right)}{p_{K}\left(V_{I, n}\right)}\left(\frac{T}{\rho c_{v}} \sum_{k=1}^{K} \frac{\alpha_{k} \partial_{T} p_{k}}{\sigma_{k}^{n} \rho_{k}}\left(\rho_{k} \delta_{k}^{n} \beta_{k, i}-\bar{a}_{k}^{2} \alpha_{k, n, i}\right)-a_{n, i}\right)=\frac{\rho c_{v} \kappa_{0}\left(V_{I, n}\right)}{p_{K}\left(V_{I, n}\right)}\left(z_{i}\left(V_{I, n}\right)-a_{n, i}\right)
\end{aligned}
$$


with $\sigma_{k}^{n}=\sigma_{k}^{n}\left(V_{I, n}\right)$ and $\delta_{k}^{n}=\delta_{k}^{n}\left(V_{I, n}\right)$ In particular, all these eigenvectors are linearly independent and span a $K-1$-dimensional subspace of $\mathbb{R}^{(K-1)+K(d+1)+1}$. The corresponding orthogonal left eigenvectors are determined by

$$
\boldsymbol{l}_{I, i}^{T}=\left(\boldsymbol{l}_{I, i, 0}^{T}, \boldsymbol{l}_{I, i, 1}^{T}, \ldots, \boldsymbol{l}_{I, i, K}^{T}, l_{I, i}\right) \quad \text { with } \quad \boldsymbol{l}_{I, i, 0}=\boldsymbol{e}_{K-1, i}, \boldsymbol{l}_{I, i, k}=\mathbf{0}_{d+1}, l_{I, i}=0 .
$$

Remark 6. Whether the corresponding characteristic field is linearly degenerated depends on the choice of the interfacial velocity $\boldsymbol{V}_{I}$. For stability reasons the right eigenvectors $\boldsymbol{r}_{I, i}$ and the left eigenvectors $\boldsymbol{l}_{I, i}$ should be scaled.

Next we determine the right and left eigenvectors to the roots of the polynomial $P_{K}$.

Proposition 3. Let $\lambda \in \mathbb{R}$ be one of the existing $2 K+1$ roots of the characteristic polynomial (3.10), i.e., $p_{K}(\lambda)=0$, and let the non-resonance condition (3.18) hold true. Then the corresponding right eigenvector is determined by

$$
\boldsymbol{r}_{\lambda}^{T}=\left(\mathbf{0}_{K-1}, \boldsymbol{r}_{\lambda, 1}^{T}, \ldots, \boldsymbol{r}_{\lambda, K}^{T}, 1\right) \quad \text { with } \quad \boldsymbol{r}_{\lambda, k}^{T}=\frac{1}{\sigma_{k}^{n}(\lambda) \rho_{k}} \partial_{T} p_{k}\left(\rho_{k},-\delta_{k}^{n}(\lambda) \boldsymbol{n}^{T}\right)
$$

Proposition 4. Let $\lambda \in \mathbb{R}$ be one of the existing $2 K+1$ roots of the characteristic polynomial (3.10), i.e., $p_{K}(\lambda)=0$, and let the non-resonance condition (3.18) as well as $\lambda \neq V_{I, n}$ hold true. Then the corresponding left eigenvector is determined by $\boldsymbol{l}_{\lambda}^{T}=l_{\lambda}\left(\boldsymbol{l}_{\lambda, I}^{T}, \boldsymbol{l}_{\lambda, 1}^{T}, \ldots, \boldsymbol{l}_{\lambda, K}^{T}, 1\right)$ with

$$
\begin{aligned}
& \boldsymbol{l}_{\lambda, I}=\frac{\boldsymbol{z}(\lambda)-\boldsymbol{a}_{n}}{V_{I, n}-\lambda}=\frac{1}{V_{I, n}-\lambda}\left(\frac{T}{\rho c_{v}} \sum_{k=1}^{K} \frac{\alpha_{k} \partial_{T} p_{k}}{\rho_{k} \sigma_{k}^{n}(\lambda)}\left(\rho_{k} \delta_{k}^{n}(\lambda) \boldsymbol{\beta}_{k}-\bar{a}_{k}^{2} \boldsymbol{\alpha}_{k, n}\right)-\boldsymbol{a}_{n}\right) \\
& \boldsymbol{l}_{\lambda, k}^{T}=\frac{\alpha_{k}}{\rho c_{v} \sigma_{k}^{n}(\lambda)} T \partial_{T} p_{k}\left(\bar{a}_{k}^{2} / \rho_{k},-\delta_{k}^{n}(\lambda) \boldsymbol{n}^{T}\right) .
\end{aligned}
$$

Choosing the scaling factor

$$
l_{\lambda}^{-1}=1+\frac{T}{\rho c_{v}} \sum_{k=1}^{K} \frac{\alpha_{k}\left(\partial_{T} p_{k}\right)^{2}}{\rho_{k}\left(\sigma_{k}^{n}(\lambda)\right)^{2}}\left(\bar{a}_{k}^{2}+\left(\delta_{k}^{n}(\lambda)\right)^{2}\right)>0
$$

then $\boldsymbol{l}_{\lambda, k}^{T} \boldsymbol{r}_{\lambda, k}=1$ where $\boldsymbol{r}_{\lambda, k}$ is the corresponding right eigenvector.

Remark 7. The subvectors $\boldsymbol{l}_{\lambda, k}$ and the scaling factor $l_{\lambda}$ are identical as in [13] except for the factor $\alpha_{k}$.

Remark 8. It is still open whether the corresponding characteristic fields are genuinely nonlinear.

Finally, it remains to verify that the above right and left eigenvectors are linearly independent. This is true if all roots of the characteristic polynomial (3.10) are simple.

Theorem 2 (Existence of an eigenvector basis). Let there be $2 K+1$ simple roots $\lambda_{i}, i=1, \ldots, 2 K+1$ of the characteristic polynomial (3.10) for which the non-resonance condition (3.18) holds true and that do not coincide with $V_{I, n}$. Then the corresponding left eigenvectors $\boldsymbol{l}_{I, i}, i=1, \ldots, K-1, \boldsymbol{l}_{\lambda_{i}}, i=1, \ldots, 2 K+1, \boldsymbol{l}_{i, 0}^{k}$, $i=1, \ldots, K, k=1, \ldots, d-1$, given by (3.27), (3.29), (3.30), (3.13) and right eigenvectors $\boldsymbol{r}_{I, i}, i=1, \ldots, K-1$, $\boldsymbol{r}_{\lambda_{i}}, i=1, \ldots, 2 K+1, \boldsymbol{r}_{i, 0}^{k}, i=1, \ldots, K, k=1, \ldots, d-1$ given by (3.26), (3.28), (3.13) are orthogonal to each other. 
Proof: First of all, we rewrite the left and right eigenvectors in block matrices $\boldsymbol{L}$ and $\boldsymbol{R}$ with rows and columns containing the left eigenvectors and right eigenvectors, respectively:

$$
\boldsymbol{L}=\left(\begin{array}{ccccc}
\boldsymbol{L}_{I, 0} & & & & \\
\boldsymbol{L}_{I} & \boldsymbol{L}_{1} & \ldots & \boldsymbol{L}_{K} & \boldsymbol{l} \\
& \boldsymbol{L}_{1}^{d} & & & \\
& & \ddots & & \\
& & & \boldsymbol{L}_{K}^{d}
\end{array}\right), \boldsymbol{R}=\left(\begin{array}{ccccc}
\boldsymbol{R}_{I, 0} & & & & \\
\boldsymbol{R}_{I, 1} & \boldsymbol{R}_{1} & \boldsymbol{R}_{1}^{d} & & \\
\vdots & \vdots & & \ddots & \\
\boldsymbol{R}_{I, K} & \boldsymbol{R}_{K} & & & \boldsymbol{R}_{K}^{d} \\
\boldsymbol{r}_{I}^{T} & \boldsymbol{r}^{T} & & &
\end{array}\right)
$$

with block matrices

$$
\begin{aligned}
& \boldsymbol{R}_{I, 0} \stackrel{(3.26 a)}{=}\left(\boldsymbol{r}_{I, 1,0}, \ldots, \boldsymbol{r}_{I, K-1,0}\right), \boldsymbol{R}_{I, k} \stackrel{(3.26 b)}{=}\left(\boldsymbol{r}_{I, 1, k}, \ldots, \boldsymbol{r}_{I, K-1, k}\right), \boldsymbol{r}_{I} \stackrel{(3.26 c)}{=}\left(r_{I, 1}, \ldots, r_{I, K-1}\right)^{T}, \\
& \boldsymbol{R}_{k} \stackrel{(3.28)}{=}\left(\boldsymbol{r}_{\lambda_{1}}, \ldots, \boldsymbol{r}_{\lambda_{2 K+1}}\right), \boldsymbol{R}_{k}^{d} \stackrel{(3.13)}{=}\left(\left(0, \boldsymbol{t}_{1}^{T}\right), \ldots,\left(0, \boldsymbol{t}_{d-1}^{T}\right), \boldsymbol{r} \stackrel{(3.28)}{=} \mathbf{1}_{2 K+1}\right.
\end{aligned}
$$

as well as

$$
\begin{aligned}
& \boldsymbol{L}_{I, 0}^{T} \stackrel{(3.27)}{=}\left(\boldsymbol{l}_{I, 1,0}, \ldots, \boldsymbol{l}_{I, K-1,0}\right), \boldsymbol{L}_{I}^{T} \stackrel{(3.29)}{=}\left(l_{\lambda_{1}} \boldsymbol{l}_{\lambda_{1}, I}, \ldots, \boldsymbol{l}_{\lambda_{2 K+1}} \boldsymbol{l}_{\lambda_{2 K+1}, I}\right), \\
& \boldsymbol{L}_{k}^{T} \stackrel{(3.30)}{=}\left(l_{\lambda_{1}} \boldsymbol{l}_{\lambda_{1}, k}, \ldots, l_{\lambda_{2 K+1}} \boldsymbol{l}_{\lambda_{2 K+1}, k}\right), \boldsymbol{L}_{k}^{d} \stackrel{(3.13)}{=}\left(\boldsymbol{R}_{k}^{d}\right)^{T}, \boldsymbol{l}^{T} \stackrel{(3.31)}{=}\left(l_{\lambda_{1}}, \ldots, l_{\lambda_{2 K+1}}\right)^{T},
\end{aligned}
$$

for $k=1, \ldots, K$. First of all, we note that for $k=1, \ldots, K$ the vectors

$$
\overline{\boldsymbol{r}}_{i, 0}^{k}=(\underbrace{\mathbf{0}_{d+1}^{T}, \ldots, \mathbf{0}_{d+1}^{T}}_{i-1},\left(0, \boldsymbol{t}_{k}^{T}\right), \underbrace{\mathbf{0}_{d+1}^{T}, \ldots, \mathbf{0}_{d+1}^{T}}_{K-i}, 0)^{T}, \quad i=1, \ldots, d-1
$$

are $d-1$ linearly independent right eigenvectors to the $(d-1)$-multiple eigenvalue $v_{k, n}$ of the matrix $\overline{\boldsymbol{B}}_{n}$ defined by (3.9). Since by assumption all roots $\lambda_{i}, i=1, \ldots, 2 K+1$, of the characteristic polynomial $p_{K}$ defined by (3.10) are distinct, then the vectors

$$
\overline{\boldsymbol{r}}_{\lambda_{i}}=\left(\boldsymbol{r}_{\lambda_{i}, 1}^{T}, \ldots, \boldsymbol{r}_{\lambda_{i}, K}^{T}, 1\right)^{T}
$$

are linearly independent right eigenvectors of the matrix $\overline{\boldsymbol{B}}_{n}$. Note that these eigenvectors coincide for multiple roots. Furthermore, the eigenvectors $\overline{\boldsymbol{r}}_{i, 0}^{k}$ and $\overline{\boldsymbol{r}}_{\lambda_{i}}$ are linearly independent as can be directly concluded from the matrices $\boldsymbol{R}_{k}$ and $\boldsymbol{R}_{k}^{d}$. Obviously, the extended vectors $\boldsymbol{r}_{i, 0}^{k}=\left(\mathbf{0}_{K-1}^{T},\left(\overline{\boldsymbol{r}}_{i, 0}^{k}\right)^{T}\right)^{T}$ and $\boldsymbol{r}_{\lambda_{i}}=\left(\mathbf{0}_{K-1}^{T}, \overline{\boldsymbol{r}}_{\lambda_{i}}^{T}\right)^{T}$ are linearly independent and are linearly independent to the right eigenvectors $\boldsymbol{r}_{I, i}, i=1, \ldots, K-1$. Thus, these vectors form a right eigenvector basis to the matrix $\overline{\boldsymbol{B}}_{n}$, i.e., the matrix $\boldsymbol{R}$ is invertible. Then the matrix $\boldsymbol{L}$ must coincide with the inverse $\boldsymbol{R}^{-1}$ except for a scaling of the rows, i.e., $\boldsymbol{L}=\boldsymbol{S} \boldsymbol{R}$ with $\boldsymbol{S}=\operatorname{diag}\left(\boldsymbol{s}^{I}, \boldsymbol{s}^{\lambda}, \boldsymbol{s}^{1}, \ldots, \boldsymbol{s}^{K}\right)$, $\boldsymbol{s}^{I}=\left(s_{I, 1}, \ldots, s_{I, K-1}\right)^{T}, \boldsymbol{s}^{\lambda}=\left(s_{\lambda_{1}}, \ldots, s_{\lambda_{2 K+1}}\right), \boldsymbol{s}^{k}=\left(s_{1,0}^{k}, \ldots, s_{d-1,0}^{k}\right)$. The scaling factors are determined by the orthogonality conditions $s_{I, i}:=\boldsymbol{r}_{I, i} \cdot \boldsymbol{l}_{I, i}, s_{\lambda_{i}}:=\boldsymbol{r}_{\lambda_{i}} \cdot \boldsymbol{l}_{\lambda_{i}}$ and $s_{i, 0}^{k}:=\boldsymbol{r}_{i, 0}^{k} \cdot \boldsymbol{l}_{i, 0}^{k}$. By definition of the left and right eigenvectors it can be verified that $\boldsymbol{S}=\boldsymbol{I}$. Thus we conclude $\boldsymbol{L} \boldsymbol{R}=\boldsymbol{I}$, i.e., the assertion holds.

Finally we conclude from Theorems 1 and 2

Conclusion 1 (Hyperbolicity). Let the assumptions of Theorems 1 and 2 hold true. Then the non-reacting homogeneous class-II model (1.2), (1.8) is hyperbolic for simple mixtures. 


\section{ThermodynamicAl PROPERTIES: 2ND LAW OF THERMODYNAMICS}

From a physical point of view, a model is admissible if it is in agreement with the principles of thermodynamics. For this purpose, we have to verify that the entropy of the mixture defined by

$$
\rho \tilde{s}=\sum_{k=1}^{K} \alpha_{k} \rho_{k} s_{k}\left(\rho_{i}, \tilde{T}\right)=\rho \tilde{s}\left(\rho e, \rho_{1}, \ldots, \rho_{K}, \alpha_{1}, \ldots, \alpha_{K-1}\right)
$$

is a concave function. Furthermore, it must be consistent with the 2nd law of thermodynamics, i.e., the entropy production terms must be non-negative. This will provide us with admissibility criteria for the interfacial pressures and interfacial velocity as well as the relaxation terms.

\subsection{Thermodynamic stability}

In case of the full Baer-Nunziato model where no equilibrium is assumed we have proven that the mixture entropy $\rho s$ is a (non-strictly) concave function of the quantities $\boldsymbol{u}:=\left(\boldsymbol{\alpha}, \alpha_{1} \boldsymbol{u}_{1}^{T}, \ldots, \alpha_{K} \boldsymbol{u}_{K}^{T}\right)^{T}$ with $\boldsymbol{u}_{k}:=$ $\left(\rho_{k}, \rho_{k} \boldsymbol{v}_{k}^{T}, \rho_{k} E_{k}\right)^{T}$ provided that the phasic energies $e_{k}$ are convex functions of the specific volume $\tau_{k}:=1 / \rho_{k}$ and the specific entropy $s_{k}$, see [23].

A similar result can be verified here if the volume fractions are constant.

Theorem 3. (Concavity of entropy function) Let the temperature $T$, the densities $\rho_{k}$ and the volume fractions $\alpha_{k}$ be positive. Furthermore, we assume that $\mu_{k}, c_{v, k}$ and $\partial_{\rho_{k}} \mu_{k}, k=1, \ldots, K$, do not vanish, i.e.,

$$
\mu_{k} \neq 0, \quad c_{v, k} \neq 0, \quad \partial_{\rho_{k}} \mu_{k} \neq 0, \quad k=1, \ldots, K .
$$

Then for fixed composition of the mixture, i.e., the volume fractions $\alpha_{k}$ are constants, the entropy defined by (4.1) becomes $\rho s=\rho \tilde{s}\left(\rho e, \rho_{1}, \ldots, \rho_{K}\right)$ and is strictly concave, if and only if

$$
\rho c_{v}>0 \quad \text { and } \quad \partial_{\rho_{k}} \mu_{k}>0, \quad k=1, \ldots, K .
$$

Proof: We will verify that the Hessian $\boldsymbol{M}=\left(\begin{array}{cc}a & \boldsymbol{a}^{T} \\ \boldsymbol{a} & \boldsymbol{A}\end{array}\right)$ of $-\rho \tilde{s}$ with

$$
a:=-\partial_{\rho e, \rho e}(\rho \tilde{s}), \quad a_{k}:=-\partial_{\rho e, \rho_{k}}(\rho \tilde{s}), k=1, \ldots, K, \quad a_{k, j}:=-\partial_{\rho_{k}, \rho_{j}}(\rho \tilde{s}), k, j=1, \ldots, K
$$

is positive definite. For this purpose we compute the derivatives of the temperature $T=\tilde{T}\left(\rho e, \rho_{1}, \ldots, \rho_{K}\right)$ using the relations (2.1) for the phasic quantities and (1.1)

$$
\partial_{\rho e} \tilde{T}=\frac{1}{\rho c_{v}}, \quad \partial_{\rho_{k}} \tilde{T}=-\frac{1}{\rho c_{v}} \alpha_{k}\left(\mu_{k}-T \partial_{T} \mu_{k}\right), k=1, \ldots, K .
$$

For the derivatives of the entropy we then obtain

$$
\partial_{\rho e} \rho \tilde{s}=\frac{1}{\tilde{T}}, \quad \partial_{\rho_{k}} \rho \tilde{s}=-\frac{1}{\tilde{T}} \alpha_{k} \mu_{k} .
$$

Thus, the coefficients can be rewritten as

$$
a=-\partial_{\rho e}\left(\frac{1}{T}\right), a_{k}=-\partial_{\rho_{k}}\left(\frac{1}{T}\right), a_{k, j}=\partial_{\rho_{j}}\left(\frac{\alpha_{k} \mu_{k}}{T}\right) .
$$

Using the derivatives (4.5) of the temperature we then can compute the second derivatives of the entropy

$$
a=1 /\left(T^{2} \rho c_{v}\right), \quad a_{k}=-\alpha_{k}\left(\mu_{k}-T \partial_{T} \mu_{k}\right) a, \quad a_{k, j}=\delta_{k, j} \alpha_{k} \partial_{\rho_{k}} \mu_{k} / T+a_{k} a_{j} / a .
$$


The matrix $\boldsymbol{M}$ can now be rewritten as

$$
\boldsymbol{A}=\boldsymbol{D}_{A}+\boldsymbol{a} \boldsymbol{a}^{T} / a \quad \text { with } \quad \boldsymbol{D}_{A}:=T^{-1} \operatorname{diag}\left(\alpha_{1} \partial_{\rho_{1}} \mu_{1}, \ldots, \alpha_{K} \partial_{\rho_{K}} \mu_{K}\right)
$$

By assumption (4.2) we can now symmetrize the matrix:

$$
\boldsymbol{L} \boldsymbol{M} \boldsymbol{L}^{T}=\left(\begin{array}{cc}
a & \mathbf{0}^{T} \\
\mathbf{0} & \boldsymbol{A}-\boldsymbol{a} \boldsymbol{a}^{T} / a
\end{array}\right)=\left(\begin{array}{cc}
a & \mathbf{0}^{T} \\
\mathbf{0} & \boldsymbol{D}_{A}
\end{array}\right), \quad \boldsymbol{L}:=\left(\begin{array}{cc}
1 & \mathbf{0}^{T} \\
-\boldsymbol{a} / a & \boldsymbol{I}
\end{array}\right)
$$

Since $\boldsymbol{D}_{A}$ is a diagonal matrix, the matrix $\boldsymbol{M}$ is positive definite if and only if (4.3) holds.

The proof is in analogy to the class-II model of Bothe and Dreyer [4]. It verifies their result in case of a simple mixture provided that the conditions (4.3) hold true. For a stiffened gas EoS and a van der Waals Eos the constraints on the material parameters have been derived from the conditions (4.3) in [13].

For the general case of non-constant volume fractions a similar result does not hold.

Theorem 4. (Indefiniteness of entropy function) Let the temperature $T$, the densities $\rho_{k}$ and the volume fractions $\alpha_{k}$ be positive. Furthermore, we assume that $\mu_{k}, c_{v, k}$ and $\partial_{\rho_{k}} \mu_{k} . k=1, \ldots, K$, do not vanish, Then the Hessian of the entropy $\rho s=\rho \tilde{s}\left(\rho e, \rho_{1}, \ldots, \rho_{K}, \alpha_{1}, \ldots, \alpha_{K-1}\right)$ defined by (4.1) is indefinite and, thus, the entropy is neither concave nor convex.

The proof is similar to the proof of Theorem 3 where the symmetrization finally results in an indefinite matrix.

Remark 9. Volume fractions might be locally constant if the flow field is locally frozen. For instance, in case of a mixture of gases this might occur whenever chemical reactions take place on a much larger time scale than transport phenomena. Typically, this is characterized by Damköhler numbers.

\subsection{Entropy production}

According to the 2nd law of thermodynamics we have to verify that the entropy production is non-negative. For this purpose we derive from the evolution equations (1.2), (1.8) and (1.11) the entropy balance

$$
\partial_{t}(\rho s)+\nabla \cdot(\rho s \boldsymbol{v}+\boldsymbol{\Phi})=\zeta
$$

The entropy flux $\boldsymbol{\Phi}$ is determined by

$$
\boldsymbol{\Phi}:=\frac{1}{T}\left(\boldsymbol{q}-\sum_{k=1}^{K} \alpha_{k}\left(\rho_{k} e_{k}+p_{k}\right)\left(\boldsymbol{v}-\boldsymbol{v}_{k}\right)+\sum_{k=1}^{K} \alpha_{k} \rho_{k} \mu_{k}\left(\boldsymbol{v}-\boldsymbol{v}_{k}\right)\right) .
$$

Following [4] the entropy production

$$
\zeta:=\zeta_{\text {interface }}+\zeta_{\text {diff,nonreact }}+\zeta_{\text {diff,react }}+\zeta_{\text {chem }}+\zeta_{\text {vol }}
$$


is composed of different additive contributions corresponding to diffusion flux with respect to non-reacting and reacting mixtures as well as chemical reaction kinetics, respectively:

$$
\begin{aligned}
& \zeta_{\text {interface }}:=\frac{1}{T} \sum_{k=1}^{K}\left(\sum_{l=1}^{K} P_{k, l}\left(\boldsymbol{v}_{k}-\boldsymbol{V}_{I}\right) \cdot \nabla \alpha_{l}+p_{k}\left(\boldsymbol{v}_{k}-\boldsymbol{V}_{I}\right) \cdot \nabla \alpha_{k}\right)=: \sum_{k=1}^{K} \zeta_{\text {interface }, k}, \\
& \zeta_{\text {diff,nonreact }}:=\frac{1}{T} \sum_{k=1}^{K}\left(\boldsymbol{v}-\boldsymbol{v}_{k}\right) \cdot \boldsymbol{S}_{\alpha \rho \boldsymbol{v}, k} \\
& \zeta_{\text {diff,react }}:=-\sum_{k=1}^{K}\left(\boldsymbol{v}-\boldsymbol{v}_{k}\right) \cdot \frac{1}{T}\left(\frac{1}{2} S_{\alpha \rho, k}\left(\boldsymbol{v}-\boldsymbol{v}_{k}\right)+S_{\alpha \rho, k} \boldsymbol{v}_{k}\right) \\
& \zeta_{\text {chem }}:=-\frac{1}{T} \sum_{k=1}^{K} S_{\alpha \rho, k} \mu_{k} \\
& \zeta_{\text {vol }}:=\frac{1}{T} \sum_{k=1}^{K} p_{k} S_{\alpha, k} .
\end{aligned}
$$

Furthermore, if we add the energy productions due to diffusion, chemistry and volume fractions we obtain the total entropy production due to relaxation

$$
\zeta_{\text {relax }}:=\frac{1}{T} \sum_{k=1}^{K}\left(p_{k} S_{\alpha, k}-\boldsymbol{v}_{k} \cdot \boldsymbol{S}_{\alpha \rho \boldsymbol{v}, k}+\left(u_{k}-\mu_{k}\right) S_{\alpha \rho, k}\right) .
$$

This term coincides with the entropy production term (neglecting heat conduction and viscosity) due to relaxation in [23] except for the energy relaxation term $S_{\alpha \rho E, k}$ that is not present here because of temperature equilibrium.

In the subsequent sections we will give sufficient conditions that ensure thermodynamical compatibility. Since the results coincide with those derived in [23] in case of a full non-equilibrium model we omit the proofs.

To investigate the admissibility of the production terms $\zeta_{\text {interface, },}$ we first make use of the assumptions $(1.5)_{1}$ and $(1.5)_{2}$ for the interfacial pressures. Then these terms become

$$
\zeta_{\text {interface }, k}=\frac{1}{T} \sum_{l=1, l \neq k}^{K}\left(P_{k, l}-p_{k}\right)\left(\boldsymbol{v}_{k}-\boldsymbol{V}_{I}\right) \cdot \nabla \alpha_{l} .
$$

Obviously, we cannot control the sign of $\zeta_{\text {interface, }, k}$. According to the conservation constraints $(1.5)_{2}$ all $P_{k, l}$, $k \neq l$, are coupled. Therefore we determine the interfacial pressures $P_{k, l}$ and the interfacial velocity $\boldsymbol{V}_{I}$ such that the sum $\zeta_{\text {interface }}=\sum_{k=1}^{K} \zeta_{\text {interface }, k}$ vanishes.

Theorem 5. (Entropy production due to interfacial states) Let the assumptions $(1.5)_{1}$ and $(1.5)_{2}$ hold true. If the temperature $T$ is positive, then for any convex combination

$$
\boldsymbol{V}_{I}=\sum_{k=1}^{K} \beta_{k} \boldsymbol{v}_{k}, \quad \beta_{k} \in[0,1], \quad \sum_{k=1}^{K} \beta_{k}=1 .
$$

for the interfacial velocity $\boldsymbol{V}_{I}$ there uniquely exist interfacial pressures

$$
P_{k, l}=\beta_{k} p_{l}+p_{k} \sum_{j=1, \neq k}^{K} \beta_{j}=\beta_{k} p_{l}+\left(1-\beta_{k}\right) p_{k}, \quad k \neq l
$$


such that the production term $\zeta_{\text {interface }}=\sum_{k=1}^{K} \zeta_{\text {interface, } k}$ vanishes. In particular, the interfacial pressures are all positive and it holds

$$
P_{I}=\sum_{k=1}^{K} p_{k} \sum_{j=1, \neq k}^{K} \beta_{j}=\sum_{k=1}^{K}\left(1-\beta_{k}\right) p_{k}
$$

The proof is in complete analogy to the proof of Theorem 6 in [23] in case of thermal non-equilibrium, where we replace the phasic temperatures $T_{k}$ by the mixture temperature $T$.

The ansatz of a convex combination (4.16) for the interfacial velocity $\boldsymbol{V}_{I}$ is motivated by Gallouët et al. [10] and Hérard [14] for a two-phase and a three-phase model, respectively. A similar result is also available for a multi-component flow model of barotropic fluids, cf. [15]. Since the interfacial pressures $P_{k, l}$ depend on (4.16), the 2nd law of thermodynamics does not uniquely characterize the interfacial velocity. There are several options discussed in the literature for two-component and three-component models, cf. [10, 14, 24].

Next we consider the entropy production due to the relaxation processes. For a single component this term is determined by

$$
\zeta_{\text {relax }, k} \equiv S_{\alpha \rho s, k}=\frac{1}{T}\left(p_{k} S_{\alpha, k}+\left(u_{k}-\mu_{k}\right) S_{\alpha \rho, k}-\boldsymbol{v}_{k} \cdot \boldsymbol{S}_{\alpha \rho \boldsymbol{v}, k}\right)
$$

where the chemical potential of component $k$ can be written as $\mu_{k}=e_{k}+p_{k} / \rho_{k}-T_{k} s_{k}$. In addition to the conservation constraints (1.7) the relaxation terms (1.6) have to satisfy the sufficient or the necessary condition

$$
S_{\alpha \rho s, k} \geq 0, k=1, \ldots, K \text { or } \sum_{k=1}^{K} S_{\alpha \rho s, k} \geq 0
$$

to ensure that the mixture is consistent with the 2 nd law of thermodynamics.

\subsection{Remarks on the closure of the interfacial velocity}

According to the ansatz (4.16) for the interfacial velocity we have some freedom in the choice of the parameters $\beta_{k}$. One option might be to choose them state-dependent, i.e., $\beta_{k}^{v}=\beta_{k}^{v}(\boldsymbol{w})$. As a consequence the linear field associated with the eigenvalue $\lambda=V_{I, n}$ will not be linearly degenerated, i.e., $\boldsymbol{r}_{I, i} \cdot \nabla \boldsymbol{w} V_{I, n} \neq 0$. Since the non-equilibrium model contains non-conservative products in the phasic momentum equation (1.2b), there is no way to cope with these terms in case of genuinely nonlinear fields. If fields associated to the non-conservative products only occur in linearly degenerated fields, then Riemann invariants of the associated field can be enforced and, thus, the exact solution of the Riemann problem exists as has been verified for Baer-Nunziato type models in [11]. Therefore, it is suggested in [10] and [14] to determine the interfacial velocity such that the associated field is linearly degenerated. To derive appropriate closing conditions in this case we proceed as in the thermal non-equilibrium case investigated in [23] where the ansatz of Saleh [24], Eqn. (4.3.40), in case of a two-phase mixture has been extended to multi-component mixtures. A similar result is also available for a multi-component flow model of barotropic fluids, cf. [15].

Theorem 6. Let be $\sum_{k=1}^{K} c_{k}=1$ a convex combination with constant coefficients $c_{k} \in[0,1]$. Define the coefficients $\beta_{k}$ in (4.16), i.e., $\boldsymbol{V}_{I}=\sum_{k=1}^{K} \beta_{k} \boldsymbol{v}_{k}$, as $\beta_{k}:=c_{k} \alpha_{k} \rho_{k} / \hat{\rho}$ with $\hat{\rho}:=\sum_{k=1}^{K} c_{k} \alpha_{k} \rho_{k}$. Then the characteristic field corresponding to the eigenvalue $\lambda=V_{I, n}=\sum_{k=1}^{d} n_{k} V_{I, k}$ is linearly degenerated.

Proof: By a straightforward calculation using (3.26) and (3.7a) we can verify that $\boldsymbol{r}_{I, i} \cdot \nabla \boldsymbol{w} \lambda_{I, i}$ vanishes.

Note that a similar choice of the $\beta_{k}$ coefficients has been first introduced in [12].

Finally, applying Theorem 6 in Theorem 5 the interfacial pressure and interfacial velocity are given by

$$
P_{I}=\sum_{k=1}^{K} p_{k}\left(1-c_{k} \alpha_{k} \rho_{k} / \hat{\rho}\right), \quad V_{I}=\sum_{i=1}^{K} c_{i} \alpha_{i} \rho_{i} \boldsymbol{v}_{i} / \sum_{k=1}^{K} c_{k} \alpha_{k} \rho_{k} .
$$




\section{RELAXATION MODEL}

The non-equilibrium model presented in Section 1 allows for different values for velocities, pressures and chemical potentials at the same point. Relaxation mechanisms drive these quantities into equilibrium. We distinguish mechanical and thermal relaxation for velocities and pressures resp. chemical potentials.

The relaxation terms are of major importance when dealing with interface problems, see for instance Saurel and Abgrall [25] or Lallemand et al. [18] for mechanical relaxation terms and Métayer et al. [20] for pressure, temperature and Gibbs free energy relaxation terms. Typically, it is assumed that pressure and velocity relax instantaneously, see [25], whereas the thermal relaxation and the relaxation of chemical potentials are much slower, see Zein [26]. For particular applications the orders of the relaxation times can be precised, for instance in the barotropic case, cf. [1].

Note that in the equilibrium model the equilibrium state is characterized by vanishing relaxation terms rather than the transient relaxation process itself. Since the equilibrium state does not depend on the order of relaxation, the relaxation times have not to be known explicitly.

In the subsequent sections we present the relaxation terms for mechanical and chemical potential relaxation. For each relaxation process we verify the constraints due to conservation (1.7) and entropy production (4.20).

\subsection{Mechanical relaxation}

The pressure relaxation implies volume variations, that induce energy variations due to the interfacial pressure work. Here we extend the pressure relaxation vector given in [25] for a two-phase model according to [26] by introducing a pressure average that we choose as the mixture pressure. The pressure relaxation terms then read

$$
S_{\alpha, k}^{p}:=\theta_{p} \alpha_{k}\left(p_{k}-p\right), S_{\alpha \rho, k}^{p}:=0, \boldsymbol{S}_{\alpha \rho \boldsymbol{v}, k}^{p}:=\mathbf{0} .
$$

Here $\theta_{p}$ denotes the pressure relaxation parameter. Similarly the velocity relaxation terms read

$$
S_{\alpha, k}^{v}=S_{\alpha \rho, k}^{v}:=0, \boldsymbol{S}_{\alpha \rho \boldsymbol{v}, k}^{v}:=\theta_{v} \alpha_{k} \rho_{k}\left(\boldsymbol{v}-\boldsymbol{v}_{k}\right)
$$

with the velocity relaxation parameter $\theta_{v}$. For more details on mechanical relaxation see Baer and Nunziato [3] or Baer [8] for two-phase models.

Obviously, the mechanical relaxation terms (5.1) and (5.2) satisfy the conservation constraints (1.7) as can be validated by the definition of the mixture pressure and the mixture velocity (1.4) and the saturation condition (1.1). Furthermore we determine by (4.19) and (5.1), (5.2) the entropy production terms

$$
S_{\alpha \rho s, k}^{v}=\theta_{v} \frac{\alpha_{k} \rho_{k}}{T}\left(\boldsymbol{v}-\boldsymbol{v}_{k}\right)^{2}, \quad S_{\alpha \rho s, k}^{p}=\theta_{p} \frac{\alpha_{k}}{T}\left(p-p_{k}\right)^{2} .
$$

This immediately implies

Theorem 7. (Entropy production due to mechanical relaxation) Let $\rho_{k} \geq 0, T>0$ and the relaxation parameters $\theta_{p}, \theta_{v} \geq 0$. Then the entropy production due to mechanical relaxation is non-negative, i.e.,

$$
S_{\alpha \rho s, k}^{p, v} \geq 0, k=1, \ldots, K \quad \text { and } \quad \sum_{k=1}^{K} S_{\alpha \rho s, k}^{p, v} \geq 0 .
$$

\subsection{Chemical potential relaxation}

Mass transfer between different phases of the same substance occurs, whenever these phases are not in chemical equilibrium. This physical matter of fact is the decisive factor for the idea to model the phase transition by relaxation of the chemical potentials. The mass transfer is driven by the difference of the chemical potentials. It is obvious, that from now on it is necessary to identify the phases. 
Exemplarily, we consider three components, i.e., $K=3$, with water vapor $(k=1)$, liquid water $(k=2)$ and inert gas $(k=3)$. Then the vector for relaxation of chemical potentials is given by

$$
\begin{aligned}
& S_{\alpha, 1}^{\mu}:=\theta_{\mu} \frac{\dot{m}}{\varrho_{1}}, S_{\alpha \rho, 1}^{\mu}:=\theta_{\mu} \dot{m}, \boldsymbol{S}_{\alpha \rho \boldsymbol{v}, 1}^{\mu}:=\theta_{\mu} \dot{m} \hat{\boldsymbol{v}}, \\
& S_{\alpha, 2}^{\mu}:=\theta_{\mu} \frac{\dot{m}}{\varrho_{2}}, S_{\alpha \rho, 2}^{\mu}:=-\theta_{\mu} \dot{m}, \boldsymbol{S}_{\alpha \rho \boldsymbol{v}, 2}^{\mu}:=-\theta_{\mu} \dot{m} \hat{\boldsymbol{v}}, \\
& S_{\alpha, 3}^{\mu}:=-\theta_{\mu} \dot{m}\left(\frac{1}{\varrho_{1}}+\frac{1}{\varrho_{2}}\right), S_{\alpha \rho, 3}^{\mu}:=0, \boldsymbol{S}_{\alpha \rho \boldsymbol{v}, 3}^{\mu}:=\mathbf{0},
\end{aligned}
$$

with the relaxation parameter $\theta_{\mu}$. Formulas for the parameters $\varrho_{1}, \varrho_{2}$ can be found in [26,27]. Similar to the interfacial velocity (4.16) we choose for the velocity $\hat{\boldsymbol{v}}$ a convex combination

$$
\hat{\boldsymbol{v}}=\sum_{k=1}^{K} \beta_{k}^{v} \boldsymbol{v}_{k}, \quad \beta_{k}^{v} \in[0,1], \quad \sum_{k=1}^{K} \beta_{k}^{v}=1
$$

For details on the physics see the book of Müller and Müller [22]. Again we note that the conservation constraints (1.7) are satisfied. Furthermore the entropy production terms can be determined by (4.19) and (5.5)

$$
\begin{aligned}
S_{\alpha \rho s, 1}^{\mu} & =\theta_{\mu} \dot{m} \frac{1}{T}\left(\frac{1}{2}\left(\hat{\boldsymbol{v}}-\boldsymbol{v}_{1}\right)^{2}-\frac{1}{2} \hat{\boldsymbol{v}}^{2}-\mu_{1}+\frac{p_{1}}{\varrho_{1}}\right), \\
S_{\alpha \rho s, 2}^{\mu} & =-\theta_{\mu} \dot{m} \frac{1}{T}\left(\frac{1}{2}\left(\hat{\boldsymbol{v}}-\boldsymbol{v}_{2}\right)^{2}-\frac{1}{2} \hat{\boldsymbol{v}}^{2}-\mu_{2}-\frac{p_{2}}{\varrho_{2}}\right), \\
S_{\alpha \rho s, 3}^{\mu} & =\theta_{\mu} \dot{m} \frac{1}{T}\left(-p_{3}\left(\frac{1}{\varrho_{1}}+\frac{1}{\varrho_{2}}\right)\right) .
\end{aligned}
$$

These terms may become negative also when $\theta_{\mu}, \dot{m}$ and $T$ are non-negative. For the sum of the phasic entropy production $S_{\rho s}^{\mu}=S_{\rho s, 1}^{\mu}+S_{\rho s, 2}^{\mu}+S_{\rho s, 3}^{\mu}$ we obtain

$$
S_{\rho s}^{\mu}=\dot{m} \theta_{\mu}\left(\sum_{k=1}^{2} \frac{(-1)^{k+1}}{T}\left(\frac{1}{2}\left(\hat{\boldsymbol{v}}-\boldsymbol{v}_{k}\right)^{2}-\mu_{k}-(-1)^{k} \frac{p_{k}}{\varrho_{k}}\right)+\frac{1}{T}\left(-p_{3}\left(\frac{1}{\varrho_{1}}+\frac{1}{\varrho_{2}}\right)\right)\right) .
$$

In a homogeneous mixture velocity, pressure and temperature are in equilibrium. Therefore, the total entropy of a homogeneous mixture is determined by the sum of $\rho s$ and the non-negative mixture entropy, [21], p. 320 . The mixture entropy given by

$$
S_{M}=-\sum_{k=1,3} \alpha_{k} \rho_{k} \frac{\kappa_{b}}{m_{k}} \ln \left(\frac{\alpha_{k}}{\alpha_{1}+\alpha_{3}}\right)
$$

with $\kappa_{b}$ the Boltzmann constant and $m_{k}$ the mass of a single molecule of component $k$, see [21], p. 54, 298, 320 . Moreover, the chemical potential of the vapor phase is now given by

$$
\mu_{1}=g_{1}+\frac{\kappa_{b} T}{m_{1}} \ln \left(\frac{\alpha_{1}}{\alpha_{1}+\alpha_{3}}\right)
$$

i.e., it does not coincide with its Gibbs free energy. Note that for vanishing third component, i.e., $\alpha_{3}=0$, the chemical potential of the vapor phase reduces to the vapor Gibbs free energy. Again, the chemical potential of the liquid phase equals its Gibbs free energy, i.e., $\mu_{2}=g_{2}$. In chemical equilibrium the chemical potentials of the vapor and the liquid phase equal each other. Accordingly, the mass flux is now a function of $\mu_{2}-\mu_{1}$, i.e.,

$$
\dot{m}=a\left(\mu_{2}-\mu_{1}\right)
$$


with $a \geq 0$ that again is an agreement with the kinetic relation in [7].

For a homogeneous mixture the entropy production (5.8) reduces to

$$
S_{\rho s}^{\mu}=\frac{1}{T} \dot{m} \theta_{\mu}\left(g_{2}-g_{1}\right)=\frac{1}{T} \dot{m} \theta_{\mu}\left(\mu_{2}-\mu_{1}+\frac{\kappa_{b} T}{m_{1}} \ln \left(\frac{\alpha_{1}}{\alpha_{1}+\alpha_{3}}\right)\right) .
$$

We determine the entropy production of the mixture entropy $S_{M}$

$$
S_{S_{M}}^{\mu}=-\frac{1}{T} \dot{m} \theta_{\mu} \frac{\kappa_{b} T}{m_{1}} \ln \left(\frac{\alpha_{1}}{\alpha_{1}+\alpha_{3}}\right) .
$$

Then the total entropy production is given by

$$
S_{\rho s}^{\mu}+S_{S_{M}}^{\mu}=\frac{1}{T} \dot{m} \theta_{\mu}\left(\mu_{2}-\mu_{1}\right) .
$$

Thus we conclude with the following theorem.

Theorem 8. (Entropy production due to relaxation of chemical potentials) For a homogeneous mixture the total entropy production is non-negative, i.e.,

$$
S_{\rho s}^{\mu}+S_{S_{M}}^{\mu} \geq 0
$$

if the relaxation parameter $\theta_{\mu}$ as well as the temperature $T$ are positive.

Remark 10. For a pure liquid-vapor flow the chemical potentials and Gibbs free energies coincide and the above calculations simplify. In analogy to the above non-homogeneous case we obtain:

For a homogeneous liquid-vapor mixture the entropy production is non-negative, i.e.,

$$
S_{\rho s}^{\mu} \geq 0
$$

if the relaxation parameter $\theta_{\mu}$ as well as the temperature $T$ are positive.

Remark 11. We emphasize that according to the saturation condition (1.1) the above three-component mixture of water vapor, liquid water and inert gas is immiscible. There is work by other authors [2, 19] who consider a mixed type model where the gas is miscible with the vapor but there is no mass transfer between either the gas and the vapor or the gas and the liquid. In this setting the vapor and the gas occupy the same volume, i.e., $\alpha_{1}=\alpha_{3}$. As a consequence, thermodynamical equilibrium corresponds to Dalton's law of the gas and vapor, i.e., $p_{2}=p_{1}+p_{3}$, whereas in our immiscible setting it holds $p_{1}=p_{2}=p_{3}$.

\section{Conclusion}

In the present work we discussed some properties of the class-II multi-component model of Baer-Nunziato type neglecting viscosity and heat conduction. The model could be verified to be hyperbolic, i.e., all eigenvalues are real and there exists a family of linearly independent eigenvectors provided that the non-resonance condition is satisfied, all eigenvalues not corresponding to the linear fields are simple and differ from the characteristic speeds of the linear fields and none of the components of the mixture vanishes.

The main interest was on the derivation of closure conditions for the relaxation model as well as the interfacial pressures and the interfacial velocity. These were set up by verifying the second law of thermodynamics. It turned out that the pressures and the interfacial velocity can be chosen such that their contribution in the entropy law of the mixture vanishes. However, this does not characterize a unique choice for the interfacial pressures and the interfacial velocity because a physically reasonable choice of the interfacial velocities could not be derived so far in the general case of $K>2$ components. The entropy production due to mechanical relaxation could be proven to be non-negative. The entropy production due to chemical relaxation is non-negative when assuming mechanical equilibrium. 


\section{REFERENCES}

[1] A. Ambroso, C. Chalons, F. Coquel, T. Galié, E. Godlewski, P.A. Raviart, and N. Seguin. The drift-flux asymptotic limit of barotropic two-phase two-pressure models. Commun. Math. Sci., 6(2):521-529, 2008.

[2] M. Bachmann, S. Müller, P. Helluy, and H. Mathis. A simple model for cavitation with non-condensable gases. In Hyperbolic problems: theory, numerics and applications, volume 17 of Ser. Contemp. Appl. Math. CAM, pages 289--296. World Sci. Publishing, Singapore, 2012.

[3] M.R. Baer and J.W. Nunziato. A two-phase mixture theory of the deflagration-to-detonation transition (DDT) in reactive granular materials. Int. J. Multiphase Flows, 12:861-889, 1986.

[4] D. Bothe and W. Dreyer. Continuum thermodynamics of chemically reacting fluid mixtures. Acta Mech., 226:1757-1805, 2015.

[5] D. Drew. Mathematical modeling of two-phase flow. Ann. Rev. Fluid Mech., 15:261-291, 1983.

[6] D.A. Drew and S.L. Passman. Theory of Multicomponent Fluids, volume 135 of Applied Mathematical Sciences. Springer, 1999.

[7] W. Dreyer, F. Duderstadt, M. Hantke, and G. Warnecke. Bubbles in liquids with phase transition. part 1: On phase change of a single vapor bubble in liquid water. Continuum Mechanics and Thermodynamics, 24:461-483, 2012.

[8] P. Embid and M. Baer. Mathematical analysis of a two-phase continuum mixture theory. Continuum Mechanics and Thermodynamics, 4:279-312, 1992.

[9] T. Flåtten and H. Lund. Relaxation two-phase flow models and the subcharacteristic condition. Mathematical Models and Methods in Applied Sciences, 21(12):2379-2407, 2011.

[10] T. Gallouët, J.-M. Hérard, and N. Seguin. Numerical modelling of two-phase flows using the two-fluid two-pressure approach. Mathematical Models and Methods in Applied Sciences, 14(5):663-700, 2004.

[11] P. Goatin and P. LeFloch. The Riemann problem for a class of resonant hyperbolic systems of balance laws. Annales Inst. Henri Poincare, 21(6):881-902, 2004.

[12] V. Guillemaud. Modélisation et Simulation Numérique des Éqoulements Diphasiques par une Approche Bifluide à deux Pressions. PhD thesis, Aix Marseille Université, 2007. https://tel.archives-ouvertes.fr/tel-0169178.

[13] M. Hantke and S. Müller. Analysis and simulation of a new multi-component two-phase flow model with phase transitions and chemical reactions. Quarterly of Applied Mathematics, 76(2):253-287, 2018.

[14] J.-M. Hérard. A three-phase flow model. Mathematical and Computer Modelling, 45:732-755, 2007.

[15] J.-M. Hérard. A class of compressible multiphase flow models. Comptes Rendus Mathématique, 354:954-959, 2016.

[16] K. Hutter and K. Jöhnk. Continuum Methods of Physical Modeling. Springer, Heidelberg, 2004.

[17] A. Kapila, R. Menikoff, J. Bdzil, S. Son, and D. Stewart. Two-phase modelling of DDT in granular materials: Reduced equations. Phys. Fluid, 13:3002-3024, 2001.

[18] M.H. Lallemand, A. Chinnayya, and O. Le Metayer. Pressure relaxation procedures for multi-phase compressible flows. International Journal for Numerical Methods in Fluids, 49(1):1-56, 2005.

[19] Hélène Mathis. A thermodynamically consistent model of a liquid-vapor fluid with a gas. ESAIM: M2AN, 2018. DOI:10.1051/m2an/2018044.

[20] O. Le Métayer, J. Massoni, and R. Saurel. Dynamic relaxation processes in compressible multiphase flows. Application to evaporation phenomena. ESAIM: PROCEEDINGS, 40:103-123, 2013.

[21] I. Müller. Thermodynamics. Pitman, London, 1985.

[22] I. Müller and W. Müller. Fundamentals of Thermodynamics and Applications. Springer-Verlag, Berlin, 2009.

[23] S. Müller, M. Hantke, and P. Richter. Closure conditions for non-equilibrium multi-component models. Continuum Mechanics and Thermodynamics, 28(4):1157-1189, 2015.

[24] K. Saleh. Analyse et Simulation Numérique par Relaxation d'Écoulements Diphasiques Compressibles. PhD thesis, Université Pierre et Marie Curie, Paris, 2012. https://tel.archives-ouvertes.fr/tel-00761099.

[25] R. Saurel and R. Abgrall. A multiphase Godunov method for compressible multifluid and multiphase flows. J. Comput. Phys., 150(2):425-467, 1999.

[26] A. Zein. Numerical methods for multiphase mixture conservation laws with phase transition. PhD thesis, Otto-von-Guericke University, Magdeburg, 2010.

[27] A. Zein, M. Hantke, and G. Warnecke. Modeling phase transition for compressible two-phase flows applied to metastable liquids. J. Comput. Phys., 229(8):2964-2998, 2008. 